\title{
Desafios da educação em Terapia Ocupacional na América Latina para a próxima década*
}

\section{Challenges for Occupational Therapy education in Latin America for next decade}

\author{
Fátima Correa Oliver ${ }^{1}$, Marta Carvalho de Almeida ${ }^{1}$, Rosé Colom \\ Toldrá1, Sandra Maria Galheigo', Selma Lancman², Roseli \\ Esquerdo Lopes ${ }^{3}$, Rosibeth Del Carmen Muñoz Palm ${ }^{4}$
}

OLIVER, F. C.; ALMEIDA, M. C.; TOLDRA, R. C; GALHEIGO, S. M.; LANCMAN, S.; LOPES, R. E.; PALM, R. D. C. M. Desafios da educação em Terapia Ocupacional na América Latina para a próxima década. Rev. Ter. Ocup. Univ. São Paulo, v. 22, n. 3, p. 298-307, set./dez. 2011.

RESUMO: O VI Encontro Latino-americano de Escolas e Docentes de Terapia Ocupacional foi realizado em outubro de 2011, na Faculdade de Medicina da USP, de modo associado ao XII Congresso Brasileiro e IX Latino-Americano de Terapia Ocupacional, ocorrido em São Paulo. O Encontro foi organizado pela Confederación Latinoamericana de Terapeutas Ocupacionales (CLATO), a Rede Nacional de Ensino e Pesquisa em Terapia Ocupacional (RENETO) e o Departamento de Fisioterapia, Fonoaudiologia e Terapia Ocupacional da FMUSP. Estiveram presentes 160 profissionais sendo 117 brasileiros e 43 estrangeiros de 8 países latino-americanos e 2 europeus. Estiveram representadas $50 \%$ das instituições de ensino brasileiras, principalmente as da região sudeste, e 35,8\% das latino-americanas. Pesquisadores convidados realizaram exposições sobre o cenário da educação brasileira e latino-americana e os desafios colocados para a Educação Superior na região. A CLATO caracterizou a formação na área e foram realizadas mesas redondas sobre o ensino de graduação e de pós-graduação com docentes brasileiros e de países estrangeiros. Foram apresentados 35 trabalhos sob a forma de pôsteres, sendo 27 sobre ensino de graduação, 3 sobre pós-graduação e 5 sobre educação superior. Na plenária final foram sugeridas a participação de estudantes nos próximos eventos, a ampliação do encontro para dois dias e a criação de grupos de trabalho que organizarão, previamente, os debates temáticos sobre as dificuldades e potencialidades da formação de graduação e pós-graduação para o VII Encontro a se realizar na Venezuela em 2013

DESCRITORES: Terapia ocupacional/tendências; Educação superior, Instituições acadêmicas.

\footnotetext{
*Artigo em que se apresentam as discussões e debates realizados durante o VI Encontro Latino-Americano de Escolas e Docentes de Terapia Ocupacional, realizado em 10 de outubro de 2011 que contou com apoio FAPESP, CAPES, Pró-reitoria de Cultura e Extensão da USP, Comissão de Cultura e Extensão da FMUSP e Fundação Faculdade de Medicina.

1. Professora doutora, docente do Departamento de Fisioterapia, Fonoaudiologia e Terapia Ocupacional da Faculdade de Medicina da USP.

2. Professora titular, docente do Departamento de Fisioterapia, Fonoaudiologia e Terapia Ocupacional da Faculdade de Medicina da USP.

3. Professora associada, docente do Departamento de Terapia Ocupacional da Universidade Federal de São Carlos.

4. Professora doutoranda, docente do Departamento de Terapia Ocupacional da Universidade Federal do Paraná.

Endereço para correspondência: Centro de Docência e Pesquisa em Terapia Ocupacional da FMUSP, Rua Cipotânea, 51 - Cidade Universitária, 05508-900, São Paulo, SP. E-mail: fcoliver@usp.br
} 


\section{INTRODUÇÃO}

$\mathrm{D}$ esde o ano 2000, os Encontros Latinoamericanos de Escolas e Docentes de Terapia Ocupacional são realizados simultaneamente aos Congressos Latino-Americanos de Terapeutas Ocupacionais e têm tratado de temas relacionados, principalmente, ao ensino de graduação. Já foram temas de encontros anteriores o Projeto Tuning, o ensino fundamentado nas competências profissionais, os padrões mínimos para a formação de profissionais, as metodologias de ensino, dentre outros de interesse dos docentes e apropriados ao contexto de construção de uma perspectiva latino-americana para o desenvolvimento da profissão.

Nesta sexta edição do Encontro Latino-Americano de Escolas e Docentes, os temas centrais foram o ensino de graduação e de pós-graduação e seus desafios, bem como as possibilidades de intercâmbios interinstitucionais no âmbito do ensino ou da pesquisa.

O VI Encontro ocorreu na cidade de São Paulo, na Faculdade de Medicina da Universidade de São Paulo, com organização de docentes dessa universidade, da Confederación Latinoamericana de Terapeutas Ocupacionales (CLATO) e da Rede Nacional de Ensino e Pesquisa em Terapia Ocupacional (RENETO). O evento obteve apoio da Fundação de Amparo à Pesquisa do Estado de São Paulo (FAPESP), da Coordenação de Aperfeiçoamento de Pessoal de Nível Superior (CAPES), da Pró-reitoria de Cultura e Extensão da Universidade de São Paulo e da Fundação Faculdade de Medicina.

O programa do evento foi composto por conferências, mesas redondas, debates e apresentação de trabalhos. Pesquisadores e docentes convidados trouxeram, na qualidade de palestrantes, subsídios importantes para a compreensão do cenário latino-americano e seu aprofundamento na direção pretendida.

\section{CONFERÊNCIAS, PALESTRAS E APRESENTAÇÕES NAS MESAS-REDONDAS E PLENÁRIA FINAL}

Na conferência de abertura, intitulada "Perspectivas e Desafios da Educação Superior na América Latina", a Profa. Dra. Mariluce Bittar, docente da Universidade Católica Dom Bosco (GO-Brasil) e pesquisadora do Grupo de Estudos e Pesquisas sobre Políticas de Educação Superior, iniciou sua explanação situando a educação no plano dos direitos sociais e afirmando o papel do Estado no seu desenvolvimento. Fundamentando-se em dados recentes, mostrou que a educação superior na América
Latina, e especialmente em alguns de seus países, é bastante elitista e excludente, evidenciando que as reformas e acordos no marco das transformações estruturais - no campo social e da educação - não chegaram aos resultados esperados. Diante desse cenário, a docente apontou que a promoção de políticas que garantam o acesso, a permanência e o sucesso escolar e a valorização do trabalho docente são questões que devem ser enfrentadas pelo Estado e pela sociedade civil dos países latino-americanos. Para a professora, o acesso ao ensino em níveis mais elevados não é apenas uma exigência econômica, mas também de democracia e justiça social.

Os demais palestrantes se apresentaram em mesa redonda, cujo tema articulador foi "Cenários, Perspectivas e Desafios para o Ensino e a Cooperação Acadêmica em Terapia Ocupacional na América Latina". O Prof. Dr. Gil Vicente Reis de Figueiredo, professor associado da Universidade Federal de São Carlos (SP- Brasil), apresentou um amplo cenário da educação superior na América Latina, com dados sobre a evolução dos investimentos públicos no campo da educação, a oferta de vagas e o acesso da população ao ensino superior em diferentes países. O professor, que preside o PROIFES - Federação de Sindicatos de Professores de Instituições Federais de Ensino Superior (Brasil) e integra a Coordenação de Ensino Superior da IEAL - Internacional de Educação na América Latina, afirmou que, ressalvadas as particularidades nacionais, as políticas de ensino superior desenvolvidas na região na década de 1990 produziram grave deterioração da educação pública e efeitos prejudiciais a trabalho docente, como a burocratização da vida acadêmica e práticas guiadas pela competição. Dados sobre carreira e remuneração docente mostraram, ainda, expressiva precariedade do trabalho dos docentes da América Latina.

Já a professora Rosibeth Del Carmen Muñoz Palm, da Universidade Federal do Paraná (PR-Brasil) e membro da diretoria da CLATO, apresentou informações quantitativas e qualitativas sobre o ensino de terapia ocupacional na América Latina. Tomando a internacionalização como perspectiva atual das instituições de ensino superior e as diversas modalidades de cooperação, a professora destacou que a cooperação internacional pode ampliar a qualidade da formação dos terapeutas ocupacionais e o desenvolvimento da terapia ocupacional como profissão e área de produção de conhecimentos.

Enquanto debatedora, a Profa. Dra. Roseli Esquerdo Lopes, da Universidade Federal de São Carlos (UFSCar) e presidente da Rede Nacional de Ensino e Pesquisa em Terapia Ocupacional (RENETO), tomou como ponto central o desafio para articular, na terapia ocupacional latino-americana, a comunicação e a inter-relação dos 
diferentes cenários nacionais, suas perspectivas em ensino de graduação e pós-graduação e a cooperação internacional. A partir do caso brasileiro, a professora enfatizou que as perspectivas para a área como um todo dependerão de sua capacidade de avanço no âmbito da pesquisa, o que, apesar do acúmulo verificado nas últimas décadas no que diz respeito à formação de pesquisadores, requer a ampliação de cursos de pós-graduação estrito senso, o acesso ao financiamento de pesquisas e a qualificação dos periódicos científicos da área, entre outros pontos.

$\mathrm{O}$ evento prosseguiu com a divisão dos trabalhos em duas mesas redondas, que ocorreram simultaneamente: uma sobre Graduação e outra sobre Pós-Graduação estrito senso em Terapia Ocupacional.

$\mathrm{Na}$ mesa redonda sobre o ensino de graduação participaram o Prof. Dr. Marcus Vinícius Machado de Almeida, professor adjunto da Universidade Federal do Rio de Janeiro, a Profa. Erna Navarrete, da Universidade do Chile, e a Profa. Elizabeth Mengelberg, da Universidade Nacional de San Martin (Argentina). O professor Marcus Vinícius apresentou e analisou o panorama dos cursos de graduação no Brasil. Discorreu sobre as mudanças na política de educação superior no Brasil nos últimos 15 anos, que resultaram em acelerado crescimento do número de cursos de terapia ocupacional, especialmente nas universidades federais e na região Sudeste, concomitante ao fechamento de número expressivo de cursos de instituições privadas. Para ele, tal processo alterou a participação do setor público na formação dos terapeutas ocupacionais brasileiros, de modo que a universidade pública hoje se apresenta como a principal garantia de crescimento e legitimação profissional. $\mathrm{O}$ professor mostrou, ainda, que é preciso avançar nos caminhos que levam à diminuição das grandes diferenças regionais, sendo necessário manter esforços coletivos e solidários para ampliar o número de cursos, o que poderia ser realizado por meio de estratégias desenvolvidas em nível nacional e internacional.

A Profa. Elizabeth Mengelberg desenvolveu sua apresentação com ênfase no sentido e na importância da pesquisa como prática constitutiva da formação. Defendeu que no cenário latino-americano devemos desenvolver estudos ligados às idiossincrasias da região e aos contextos sociais dos países, para que os estudantes tenham oportunidade de desenvolver capacidades de interpretação, análise e síntese de informação, busca de solução de problemas e pensamento crítico. Nesse sentido, apontou que a terapia ocupacional se interessa e atua sobre as relações entre as atividades humanas e a saúde e o bemestar individual e social e que, dessa forma, as investigações de caráter qualitativo são mais apropriadas. Segundo a professora, os diferentes gêneros narrativos podem permitir que conheçamos como as pessoas apreendem, interpretam, constroem o mundo e dão sentido a suas vidas, seus mundos, sua experiência e a suas transações relativas ao mundo social, o que é essencial para o trabalho dos terapeutas ocupacionais.

AProfa. Erna Navarrete discorreu sobre a importância das práticas profissionais no contexto de formação acadêmica dos terapeutas ocupacionais, destacando a experiência da Universidade do Chile, cujo plano curricular se desenvolve em 5 anos, fundamentado nas concepções da Ciência da Ocupação. Partindo de considerações sobre o conhecimento prático, discriminou as chamadas atividades integradas no contexto de seu curso, enquanto atividades eminentemente práticas, individuais ou grupais. Estas são realizadas junto a diferentes grupos para os quais a terapia ocupacional dirige seu trabalho, têm o objetivo comum de introduzir o estudante no mundo profissional e oferecer oportunidades para que desenvolva sua identidade profissional entre outros profissionais. Tais práticas se iniciam nos primeiros níveis da formação e se tornam mais complexas na medida em que o estudante avança para os próximos níveis. Para ela, os resultados evidenciam que as práticas integradas favorecem a constituição de um sujeito capaz de identificar suas necessidades de aprendizagem, de se auto dirigir, buscar as fontes de informação que necessita, e ainda ser capaz de enriquecer o grupo no qual se insere, confrontando-se com as incertezas inerentes ao exercício profissional.

A debatedora, Profa. Dra. Cláudia Galvão (Universidade Federal da Paraíba), apresentou uma síntese das palestras, identificando pontos que, a seu ver, mereciam destaque e expunham as peculiaridades do momento atual. Assim, ressaltou os processos de reformulação e as novas perspectivas dos projetos pedagógicos em curso, que buscam ancoragem na integração com as políticas públicas de educação, saúde e assistência social; os esforços que têm sido empreendidos para implementar a integração ensinoserviço, considerando a necessária relação de troca entre a universidade e a sociedade e, ainda, os projetos que buscam produzir conexões produtivas entre as atividades de ensino, pesquisa e extensão de serviços.

$\mathrm{Na}$ mesa redonda sobre o ensino de terapia ocupacional em nível de pós-graduação estrito senso participaram os professores: Thelma Simões Matsukura, coordenadora do Programa de Pós-Graduação em Terapia Ocupacional da UFSCar - Brasil; Víctor Miranda, docente e diretor da Escola de Terapia Ocupacional da Faculdade de Saúde, Psicologia e Reabilitação da Universidade das 
Américas no Chile e docente do Mestrado em Terapia Ocupacional da Escola de Terapia Ocupacional da Universidade Andrés Bello de Santiago, Chile; Carmen Aleida Fernandez, docente do Curso de Graduação em Terapia Ocupacional e do Mestrado em Deficiência e Inclusão Social da Universidade Nacional da Colômbia; Liliana Paganizzi, docente do Curso de Terapia Ocupacional da Universidade de San Martin, Argentina; e Alejandro Guajardo, diretor do Programa de Mestrado em Terapia Ocupacional da Faculdade de Ciências e de Reabilitação da Escola de Terapia Ocupacional da Universidade Andrés Bello de Santiago, Chile e docente da Escola de Terapia Ocupacional da Universidade do Chile.

A discussão da pós-graduação estrito senso foi bastante instigante, pois tornou evidentes as diferenças nacionais no que se refere às propostas, às exigências para credenciamento de orientadores e docentes e às formas de implementação dos programas. A começar, entre os países representados na mesa redonda, apenas a Colômbia e o Brasil contam com docentes com título de doutor ministrando aulas e orientando nos programas. As experiências apresentadas apontaram para uma preocupação com a criação de quadros acadêmicos, em uma profissão ainda emergente no âmbito da pós-graduação estrito senso.

A Profa. Thelma Matsukura apresentou dados do único programa de mestrado específico em terapia ocupacional no Brasil - o Programa de Pós-Graduação em Terapia Ocupacional da Universidade Federal de São Carlos. O programa conta com duas linhas de pesquisa: "Promoção do Desenvolvimento Humano nos Contextos da Vida Diária", e "Redes Sociais e Vulnerabilidade". Trata-se de programa recente, que iniciou suas atividades em 2010 sob aprovação da Área 21 da Coordenação de Aperfeiçoamento de Pessoal de Nível Superior. O programa teve 26 alunos inscritos no processo seletivo de 2010, com 14 ingressantes e outros 41 inscritos, em 2011, com 13 ingressantes. O corpo docente é composto por 14 pesquisadores doutores, sendo 10 do núcleo permanente e 4 colaboradores. A professora afirmou que, no Brasil, existe significativa demanda por terapeutas ocupacionais pós-graduados para atender aos cursos de terapia ocupacional que entraram em funcionamento na última década, considerando que o levantamento realizado em 2008 pela RENETO contabilizou apenas cerca de 100 terapeutas ocupacionais doutores. Finalizou apontando que os inúmeros esforços para a criação e manutenção dos programas de pós-graduação são essenciais para o fortalecimento e expansão da produção do conhecimento específico de terapia ocupacional.

A Profa. Carmen Aleida Fernandez apresentou os desafios de se criar um programa de mestrado sobre as deficiências enquanto fenômeno social, envolvendo várias áreas de conhecimento e voltado a aplicações práticas e tecnológicas. O programa, Mestrado em Deficiência e Inclusão Social, foi criado conjuntamente por docentes de terapia ocupacional, fisioterapia e fonoaudiologia. Afirmou que o objetivo principal é o de promover mudanças no que diz respeito à concepção de deficiência enquanto um problema individual, perspectiva ainda hegemônica na Colômbia. O programa tem 7 anos de funcionamento e, desde 2007, é coordenado pela terapia ocupacional com o objetivo de favorecer a compreensão da complexidade da realidade social da deficiência e a preparação para a tomada de decisões em cenários políticos em processo de mudança. No contexto do próprio programa, foram produzidos livros que apresentam textos elaborados pelos estudantes. A Profa. Aleida destacou que tem havido forte investimento nas alianças com grupos de investigação interinstitucionais e também na participação em projetos sociais com projeção nacional e internacional.

O Prof. Víctor Miranda apresentou a necessidade de se criar programas de pós-graduação estrito e lato senso no Chile que, segundo ele, não têm escapado da influência da globalização. Para ele, as tendências epidemiológicas e demográficas próprias do país geram a necessidade de formar profissionais que conheçam a forma de enfrentar condições de saúde, educação e justiça que apresentam um alto componente psicossocial. Argumentou, ainda, sobre a importância de se dar ênfase aos estudos sobre qualidade de vida e desempenho ocupacional de indivíduos e grupos, que se relacionem a diferentes aspectos dos direitos e da cidadania. Advogou a criação de programas que, sem deixar de lado as raízes latino-americanas, possam contar com profissionais qualificados por meio de cooperação internacional entre países e universidades. Ressaltou os desafios para propor, realizar e debater sobre a fundamentação ideológica e conceitual da profissão, ressaltando, ainda, a necessidade de se incorporar e fortalecer as competências, atitudes e destrezas da profissão.

O Prof. Alejandro Guajardo, também do Chile, apresentou o programa sob sua direção, que tem 7 anos de existência. Inicialmente, discutiu criticamente sobre o sentido da educação no país, destacando tendências, tais como a educação como estratégia para a mobilidade social, a educação como produto de consumo, focada no mercado, e a educação para o modelo de desenvolvimento social dominante. Nesse contexto, situou os desafios de se criar um programa de pós-graduação fundamentado no princípio da continuidade entre a graduação e a pós-graduação. 
Refletiu, também, sobre as necessidades atuais de se pensar a pós-graduação em consonância com o contexto social e histórico do Chile contemporâneo, no sentido de preparar profissionais para o desenvolvimento do protagonismo e seu engajamento na resolução dos problemas sociais. Defendeu a opção por uma formação crítica, problematizadora dos fundamentos da terapia ocupacional e de seu papel social, a partir de uma postura política com foco nos direitos humanos.

A Profa. Liliana Paganizzi, docente da Universidade de San Martin, Argentina, trouxe experiência que oferece espaços abertos, flexíveis e intergeracionais de reflexão e discussão entre docentes, profissionais e estudantes de todos os níveis, que congrega 11 cursos de terapia ocupacional de universidades argentinas e diferentes associações profissionais, não apenas de terapia ocupacional. As oportunidades de reflexão, que ocorrem de maneira não muito sistematizada, são parte de uma experiência de educação não formal e têm resultado no aumento da cooperação inter e intra-universitária, ao mesmo tempo em que têm se constituem em lugar de discussão crítica e histórica da profissão. Um dos aspectos essenciais que sustenta a experiência é a promoção do diálogo com os autores e as produções próprias da terapia ocupacional, de modo a reconhecer e respeitar a construção cultural e histórica dos diferentes contextos de produção.

Durante os debates a Profa. Selma Lancman, uma das coordenadoras da mesa redonda, chamou a atenção para o fato de que, no Brasil, também há programas de pósgraduação, em Ciências da Reabilitação na Universidade de São Paulo e na Universidade Federal de Minas Gerais com orientadores de terapia ocupacional em nível de mestrado e de doutorado, programas conjuntos com as areas de fisioterapia e fonoaudiologia.

As sínteses dos debates realizados após cada mesaredonda foram relatadas na plenária final, que contou com participação expressiva e ativa da platéia. Após a apreciação do conjunto das atividades e das discussões realizadas, os participantes decidiram pela constituição de grupos de trabalho para organizar o material apresentado e promover formas de dar continuidade às discussões realizadas e de preparar os trabalhos para o VII Encontro, que acontecerá em 2013 na Venezuela.

No processo de avaliação do evento foram destacados tanto os aspectos positivos quanto as dificuldades encontradas. Avaliou-se positivamente a qualidade dos trabalhos apresentados e os aspectos organizativos do encontro. A questão das diferenças linguísticas ainda permeia a dificuldade de comunicação e a ampliação do diálogo latino-americano. Segundo alguns participantes, a maior circulação e efetiva apropriação da literatura produzida pela terapia ocupacional latino-americana é um aspecto essencial para a integração. Foi apontada como aspecto negativo a ausência de espaços para a realização de pequenos grupos de trabalho e rodas de conversa, onde os temas e as trocas interpessoais pudessem ser aprofundados. A necessidade de incluir estudantes de graduação e pós-graduação também foi assinalada para os próximos encontros.

\section{A PARTICIPAÇÃO NO EVENTO}

O evento contou com 160 participantes, sendo 117 brasileiros e 43 provenientes de outros países. Sete instituições de organização profissional estavam representadas. Havia participantes de 8 países latinoamericanos e dois europeus. Cincoenta e uma escolas de terapia ocupacional estavam presentes, sendo 29 brasileiras, 19 latino-americanas e 3 européias. Sabemos da escassez de recursos para profissionais participarem de eventos distantes de sua moradia e podemos afirmar que o encontro atingiu seu público alvo de forma satisfatória, considerando, que no Brasil existem atualmente $58^{(1)}$ cursos ativos e que, no momento da organização do evento, eram 53 os cursos de graduação na América Latina.

Conforme Tabela 1, dos 13 países da América Latina e Caribe com cursos de terapia ocupacional, estiveram presentes docentes de 7 deles: Brasil, Argentina, Bolívia, Chile, Colômbia, Peru e Venezuela e também profissional de Costa Rica. Estiveram ausentes participantes de Cuba, Panamá, Porto Rico e Uruguai, que são países em fase inicial de desenvolvimento da profissão. A ausência de colegas do México foi lamentada, dado que esse país possui um número importante de cursos de graduação.

A distribuição dos docentes participantes e das escolas por país é apresentada no Gráfico 1. Os países mais representados foram o Brasil, país sede, com 117 participantes, seguido da Argentina com 16 e, do Chile e da Colômbia, ambos com 9. Esta distribuição é proporcional ao número de escolas de terapia ocupacional.

(1) Conforme levantamento feito pelo palestrante Prof. Dr. Marcus Vinicius Machado de Almeida para sua apresentação no evento. 
OLIVER et al. Desafios da educação em Terapia. Rev. Ter. Ocup. Univ. São Paulo, v. 22, n. 3, p. 298-307, set./dez. 2011.

Tabela 1 - Representação institucional latino-americana, segundo países, escolas e participantes (exceto Brasil)

\begin{tabular}{|c|c|c|c|c|}
\hline \multicolumn{5}{|c|}{ Organização Profissional } \\
\hline \multicolumn{5}{|c|}{ Confederación Latinoamericana de Terapeutas Ocupacionales } \\
\hline País & $\begin{array}{l}\mathbf{N}^{0} \text { Total de } \\
\text { Escolas }\end{array}$ & $\begin{array}{l}\mathbf{N}^{0} \text { de Escolas } \\
\text { Presentes* }(\%)\end{array}$ & Nome das Escolas Presentes & $\mathbf{N}^{0}$ de Participantes \\
\hline \multirow{6}{*}{ ARGENTINA } & \multirow{6}{*}{11} & \multirow{6}{*}{$6(54,5 \%)$} & 1. Instituto Universitario Gran Rosario & 1 \\
\hline & & & 2.Universidad de Buenos Aires & 2 \\
\hline & & & 3.Universidad Nacional de Quilmes & 2 \\
\hline & & & 4.Universidad Nacional de San Martim & 3 \\
\hline & & & 5.Universidad Nacional del Litoral & 7 \\
\hline & & & 6.Universidade Abierta Interamericana & 1 \\
\hline BOLÍVIA** & 1 & $1(100,0 \%)$ & 1.Universidad Mayor de San Andres & 1 \\
\hline \multirow{6}{*}{ CHILE } & \multirow{6}{*}{11} & \multirow{6}{*}{$6(54,5 \%)$} & 1.Universidad Andrés Bello & 2 \\
\hline & & & 2.Universidad Austral de Chile & 2 \\
\hline & & & 3.Universidad Central de Chile & 2 \\
\hline & & & 4.Universidad de Las Americas & 1 \\
\hline & & & 5.Universidad Mayor & 1 \\
\hline & & & 6.Universidad Nacional del Chile & 1 \\
\hline \multirow{4}{*}{ COLÔMBIA } & \multirow{4}{*}{10} & \multirow{4}{*}{$4(40,0 \%)$} & 1.Escuela Colombiana de Rehabilitación & 1 \\
\hline & & & 2.Universidad de Santander & 4 \\
\hline & & & 3.Universidad del Rosário & 1 \\
\hline & & & 4.Universidad Nacional de Colombia & 3 \\
\hline PERU & 2 & $1(50,0 \%)$ & 1.Universidad Nacional Mayor de San Marcos & 3 \\
\hline VENEZUELA & 3 & $1(33,3 \%)$ & 1.Universidad Central de Venezuela & 1 \\
\hline COSTA RICA & 1 & - & Profissional não vinculado ao ensino & 1 \\
\hline CUBA & 1 & - & & - \\
\hline MÉXICO & 10 & - & & - \\
\hline PANAMÁ & 1 & - & & - \\
\hline PORTO RICO & 1 & - & & - \\
\hline URUGUAI & 1 & - & & - \\
\hline Sub-total & 53 & 19 & Total Participantes América Latina & 40 \\
\hline \multirow{2}{*}{ ESPANHA *** } & \multirow{2}{*}{-} & 1 & 1.Universidad de A Coruña & 1 \\
\hline & & 1 & 2.Universitat de Vic & 1 \\
\hline FRANÇA*** & - & 1 & 1.ADERE Paris**** & 1 \\
\hline Total & 53 & 22 & Total de Participantes & 43 \\
\hline
\end{tabular}

*Foram utilizados os dados disponíveis no Catálogo de Carreras da CLATO, divulgado em 2009.

**Até 2009, não havia curso de graduação em terapia ocupacional na Bolívia.

*** Não computado o percentual por não serem escolas latino-americanas.

****Association pour le Dévelopement, l'Enseignement et la Recherche en Ergothérapie. 
Gráfico 1 - Participantes docentes, segundo país e número de escolas

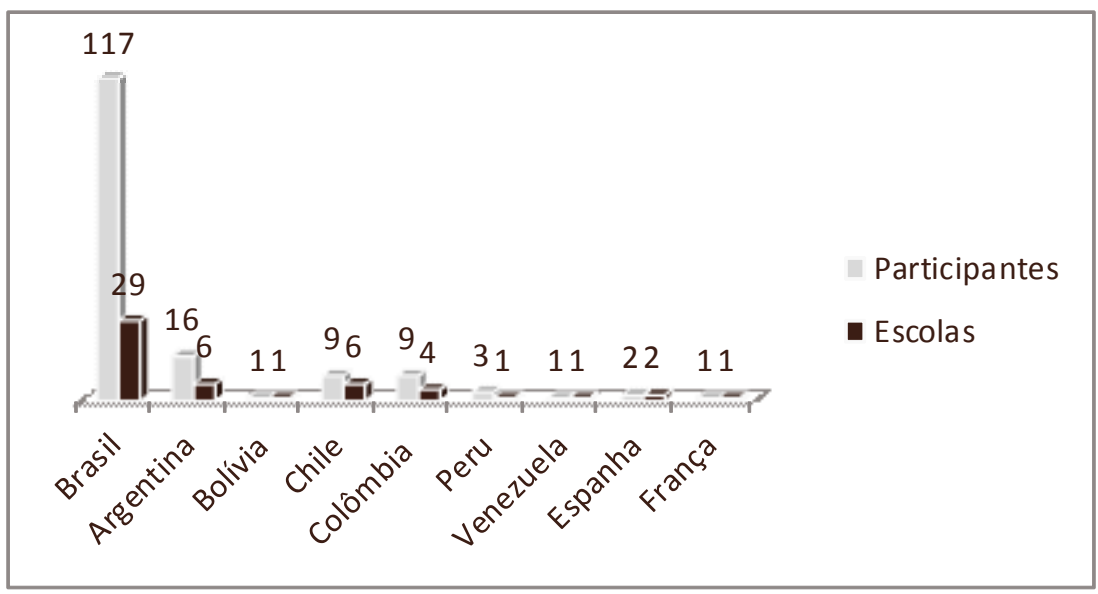

Os dados demonstram que a distribuição se mantém proporcional nos países mais representados. Assim, o país sede, o Brasil, contou com 29 (50\%) escolas presentes, seguido da Argentina e Chile, ambos com 6 escolas e Colômbia com 4.

A Tabela 2 apresenta a discriminação da participação brasileira, por organização profissional e escolas.

A distribuição dos docentes participantes e das escolas por país é apresentada no Gráfico 1. Os países mais representados foram o Brasil, país sede, com 117 participantes, seguido da Argentina com 16 e, do Chile e da Colômbia, ambos com 9. Esta distribuição é proporcional ao número de escolas de terapia ocupacional.

Dos 117 participantes brasileiros, 12 representavam organizações profissionais, 3 eram profissionais de serviços de saúde e 102 trabalhavam no ensino de terapia ocupacional, sendo a maior parte da região Sudeste, que concentra o maior número de cursos de terapia ocupacional e foi sede do evento, seguida pelo Nordeste e Sul. As regiões Centro-Oeste e Norte, que possuem o menor número de cursos e profissionais, apresentaram, conforme o esperado, um número menor de participantes, como demonstra o Gráfico 2.

Gráfico 2 - Participantes brasileiros, segundo regiões do país e número de escolas

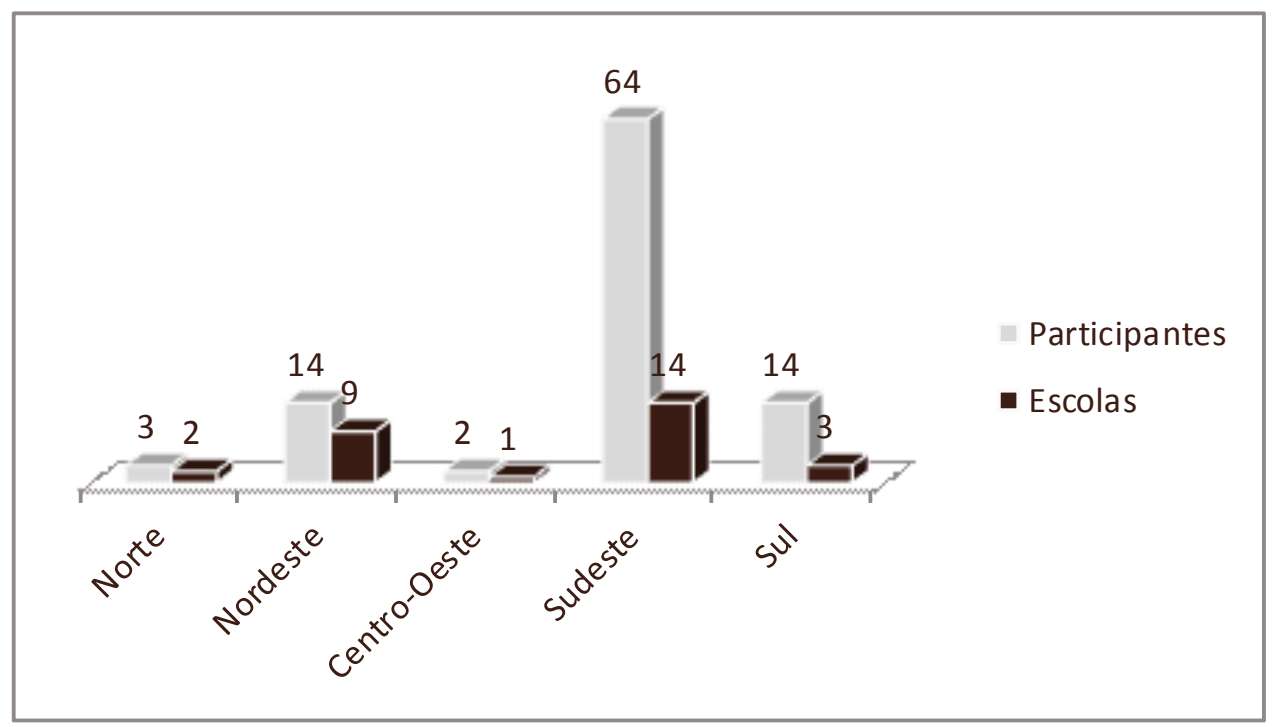


OLIVER et al. Desafios da educação em Terapia. Rev. Ter. Ocup. Univ. São Paulo, v. 22, n. 3, p. 298-307, set./dez. 2011.

Tabela 2 - Participantes brasileiros, segundo representação institucional, regiões e estados do país

\begin{tabular}{|c|c|c|c|}
\hline Entidades & $\mathbf{N}^{\mathbf{o}}$ & Regiões & Estado \\
\hline Associação Brasileira de Terapia Ocupacional & 2 & País & - \\
\hline Fórum de Professores das Instituições Federais de Ensino Superior & 1 & País & - \\
\hline Conselho Federal de Terapia Ocupacional & 1 & País & - \\
\hline Associação Cearense de Terapia Ocupacional & 3 & Nordeste & Ceará \\
\hline Associação de Terapeutas Ocupacionais do Rio Grande do Sul & 1 & Sul & Rio Grande do Sul \\
\hline Associação de Terapeutas Ocupacionais de São Paulo & 2 & Sudeste & São Paulo \\
\hline Conselho Regional de Terapia Ocupacional/CREFITO 6 & 2 & Nordeste & Ceará \\
\hline \multicolumn{4}{|l|}{ Profissionais de serviços de saúde } \\
\hline Centro de Atenção Psicossocial & 2 & Sudeste & São Paulo \\
\hline Hospital Santa Marcelina & 1 & Sudeste & São Paulo \\
\hline \multicolumn{4}{|l|}{ Instituição de Ensino Superior } \\
\hline Universidade Federal do Pará & 2 & Norte & Pará \\
\hline Universidade da Amazônia & 1 & Norte & Amazonas \\
\hline Universidade de Brasília & 2 & Centro-Oeste & Distrito Federal \\
\hline Faculdade Santa Terezinha & 2 & Nordeste & Maranhão \\
\hline Escola Baiana de Medicina e Saúde Pública & 1 & Nordeste & Bahia \\
\hline Universidade Estadual da Paraíba & 1 & Nordeste & Paraíba \\
\hline Universidade Federal da Bahia & 1 & Nordeste & Bahia \\
\hline Universidade Federal da Paraíba & 2 & Nordeste & Paraíba \\
\hline Universidade Federal de Pernambuco & 2 & Nordeste & Pernambuco \\
\hline Universidade Federal de Sergipe & 2 & Nordeste & Sergipe \\
\hline Universidade Estadual de Ciências da Saúde de Alagoas & 1 & Nordeste & Alagoas \\
\hline Universidade de Fortaleza & 2 & Nordeste & Fortaleza \\
\hline Faculdade das Ciências Médicas de Minas Gerais & 1 & Sudeste & Minas Gerais \\
\hline Faculdade de Medicina do ABC & 5 & Sudeste & São Paulo \\
\hline Instituto Federal do Rio de Janeiro & 2 & Sudeste & Rio de Janeiro \\
\hline Pontifícia Universidade Católica de Campinas & 1 & Sudeste & São Paulo \\
\hline Centro Universitário São Camilo & 1 & Sudeste & São Paulo \\
\hline Universidade Federal do Espírito Santo & 2 & Sudeste & Espírito Santo \\
\hline Universidade Federal de Minas Gerais & 2 & Sudeste & Minas Gerais \\
\hline Universidade Federal do Rio de Janeiro & 4 & Sudeste & Rio de Janeiro \\
\hline Universidade Federal de São Carlos & 11 & Sudeste & São Paulo \\
\hline Universidade Federal do Triângulo Mineiro & 1 & Sudeste & Minas Gerais \\
\hline Universidade Federal de São Paulo & 11 & Sudeste & São Paulo \\
\hline Universidade de Sorocaba & 2 & Sudeste & São Paulo \\
\hline Universidade de São Paulo & 24 & Sudeste & São Paulo \\
\hline Universidade de São Paulo - Campus Ribeirão Preto & 2 & Sudeste & São Paulo \\
\hline Universidade Federal do Paraná & 12 & Sul & Paraná \\
\hline Centro Universitário Metodista & 1 & Sul & Rio Grande do Sul \\
\hline Centro Universitário Franciscano & 1 & Sul & Rio Grande do Sul \\
\hline Total & 117 & & \\
\hline
\end{tabular}




\section{APRESENTAÇÃO DE TRABALHOS NO EVENTO}

A submissão de trabalhos para o evento foi realizada sob a forma de pôsteres institucionais para eixos temáticos, focados nos cenários, perspectivas e desafios para o ensino e a cooperação internacional em terapia ocupacional:

I. Educação Superior, História e Identidade na América Latina
II. Graduação em Terapia Ocupacional

III. Pós-Graduação Estrito Senso em Terapia Ocupacional

Dos trabalhos aceitos (35), a maioria se dedicou ao eixo da Graduação em Terapia Ocupacional, o que confirma a importância que a temática tem nas discussões da área. O Gráfico 3 traz a distribuição dos trabalhos por idioma e eixo temático.

Gráfico 3 - Trabalhos apresentados sob a forma de pôsteres, segundo idioma e eixo temático

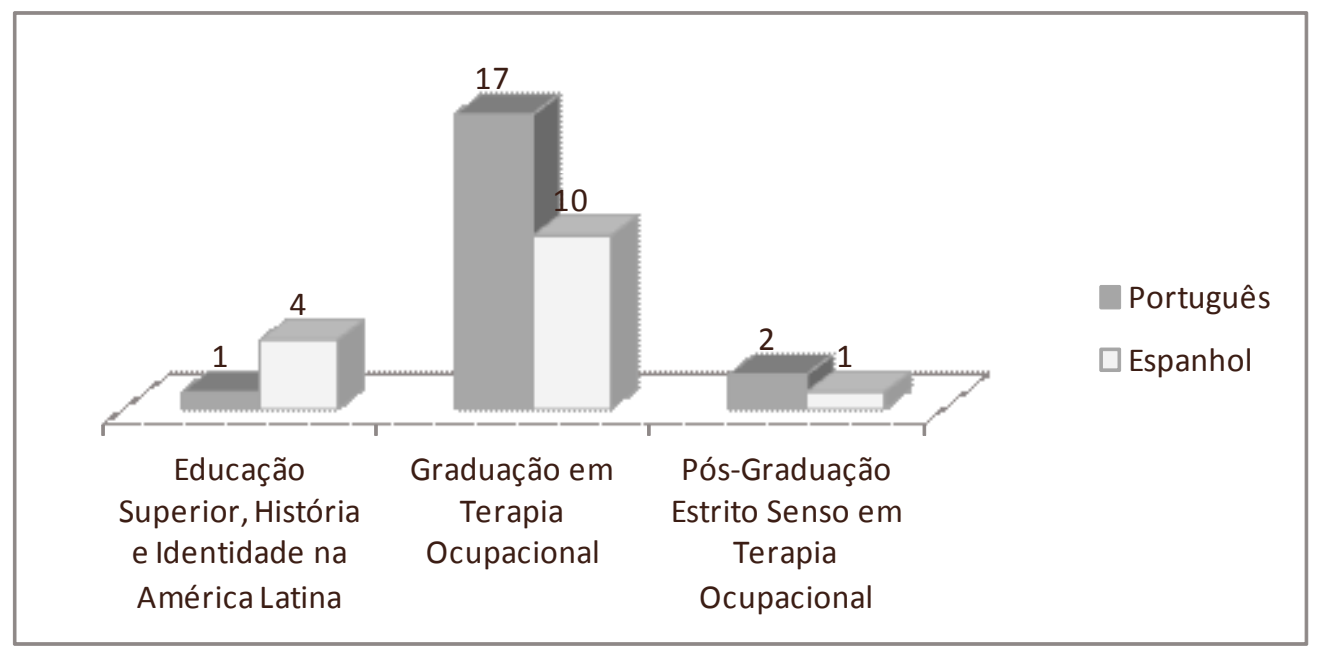

Os resumos dos trabalhos encontram-se nos Anais do VI Encontro Latino-Americano de Escolas e Docentes de Terapia Ocupacional, publicados na Revista de Terapia Ocupacional da USP, v.22, Anais (suplemento especial), 2011.

\section{CONSIDERAÇÕES FINAIS}

O VI Encontro Latino-Americano de Escolas e Docentes possibilitou a articulação docente com vistas a uma maior integração interinstitucional no âmbito do ensino de graduação e de pós-graduação, bem como da pesquisa. O número de escolas e docentes presentes foi significativo e superior aos encontros anteriores. Os apoios financeiros obtidos foram essenciais para garantir a presença de docentes e pesquisadores do Brasil e do exterior nas mesas-redondas e nos debates, o que, sem dúvida, também incrementou a participação de ouvintes nacionais e estrangeiros. Os auxílios possibilitaram, igualmente, promover o registro da história do evento ao financiar a confecção de DVD com o conteúdo das mesas redondas, conferências e demais atividades, assim como a publicação dos Anais.

As experiências apresentadas mostraram que, embora os cursos de graduação em terapia ocupacional na América Latina apresentem características comuns, acentuadas diferenças entre o Brasil e os demais países latino-americanos foram constatadas, principalmente no que se refere à pesquisa e à pós-graduação estrito senso.

O processo de produção de conhecimento na área encontra-se mais consolidado no Brasil, tendo em vista o maior número de profissionais que realizaram capacitação para a pesquisa. Ao mesmo tempo, as exigências brasileiras para criação de programas de pós-graduação e acesso a financiamento de pesquisa têm dificultado o ingresso de docentes brasileiros em programas de pós-graduação, o que pode comprometer o avanço da área no país. Quanto a esse aspecto, observa-se maior flexibilidade, principalmente no Chile e na Colômbia, o que pode contribuir para que 
a formação de docentes nesses países ocorra de maneira mais rápida. A estruturação do ensino de graduação e a organização profissional, bem como um sistema de ensino superior com maior participação de instituições públicas, que privilegiam a titulação docente favorecerão a ampliação do papel de liderança dos profissionais brasileiros na região.

O evento atingiu os objetivos propostos, permitiu o reconhecimento das identidades nacionais, que integram o universo acadêmico latino-americano, e criou alicerces importantes para futuras parcerias e para a cooperação latino-americana em vários planos, tanto do ensino de graduação como de pós-graduação e de pesquisa. Houve consenso em torno da necessidade de ampliação do evento para dois dias, o que parece também confirmar os resultados positivos do encontro.

Vale destacar também a importância da organização docente em torno da RENETO e da CLATO, o que tem possibilitado a consolidação da terapia ocupacional na região.

OLIVER, F. C.; ALMEIDA, M. C.; TOLDRA, R. C.; GALHEIGO, S. M.; LANCMAN, S.; LOPES, R. E.; PALM, R. D. C. M. Challenges for occupational therapy education in Latin America for next decade. Rev. Ter. Ocup. Univ. São Paulo, v. 22, n. 3, p. 298-307, set./dez. 2011.

\begin{abstract}
The VI Latin American Meeting of Schools and Teachers of Occupational Therapy (OT) took place in October 2011, at the Faculty of Medicine of USP, being associated with the XII Brazilian and IX Latin American Occupational Therapy Congress, held in São Paulo. The meeting was organized by the Latin American Confederation of Occupational Therapists (CLATO), the National Network of Education and Research in Occupational Therapy (RENETO) and the Department of Physical Therapy, Speech Therapy and Occupational Therapy of FMUSP. At the event 160 professionals were present among which 117 Brazilian and 43 from eight Latin American countries and two European. Fifty percent of the Brazilian educational institutions were represented, mainly from the southeast, and $35.8 \%$ of the Latin American ones. Invited researchers exhibited about the education in Brazil and Latin America and, about the challenges for higher education in the region. CLATO characterized OT education and, round tables were held about the undergraduate and graduate programs by teachers from Brazil and Latin America. Thirty-five papers were presented in the form of posters: 27 on undergraduate education, 3 on graduate education and 5 on higher education. The final meeting of the event recommended the participation of students in future events, the expansion of the event for two days, and the creation of working groups to organize in advance the thematic debates on the difficulties and potentialities of undergraduate and graduate OT Education for the VII Meeting to be held in Venezuela in 2013.
\end{abstract}

KEYWORDS: Occupational therapy/trends; Education, higher; Schools. 